\title{
Chronic pancreatitis in dogs: a retrospective study of clinical, clinicopathologic, and histopathologic findings of 61 cases
}

\author{
Brier M. Bostrom ${ }^{\mathrm{a}, \mathrm{e}}$, Panagiotis G. Xenoulis ${ }^{\mathrm{a}}$, Shelley J. Newman ${ }^{\mathrm{b}}$, Roy R. Pool ${ }^{\mathrm{c}}$, \\ Geoffrey T. Fosgate ${ }^{\mathrm{d}}$, Jörg M Steiner ${ }^{\mathrm{a},{ }^{*}}$ \\ ${ }^{a}$ Gastrointestinal Laboratory, Department of Small Animal Clinical Sciences, College of Veterinary Medicine and \\ Biomedical Sciences, Texas A\&M University, College Station, TX 77843-4474, USA \\ ${ }^{b}$ Department of Pathobiology, College of Veterinary Medicine, University of Tennessee, Knoxville, TN 37996-4542, \\ USA \\ ${ }^{c}$ Department of Pathobiology, College of Veterinary Medicine, Texas A\&M University, College Station, TX 77843- \\ 4474, USA \\ ${ }^{d}$ Department of Production Animal Studies, Faculty of Veterinary Science, University of Pretoria, Onderstepoort, \\ South Africa \\ ${ }^{e}$ Dr. Bostrom's current address is: VETMED, 20610 N. Cave Creek Rd., Phoenix, AZ 85024, USA \\ *Corresponding author. Tel: +1 9794583303. \\ E-mail address: jsteiner@,cvm.tamu.edu (J.M. Steiner)
}

\begin{abstract}
The objective of the present study was to characterize the clinical, clinicopathologic, and histopathologic findings of dogs with chronic pancreatitis. The necropsy database at Texas A\&M University was searched for reports of dogs with histologic evidence of chronic pancreatitis defined as irreversible histologic changes of the pancreas, i.e. fibrosis and atrophy. Medical records and necropsy reports were retrieved and reviewed. A reference necropsy population of 100 randomly selected dogs was used for signalment and concurrent disease comparisons. Cases were categorized as clinical or incidental chronic pancreatitis based on the presence of vomiting, decreased appetite, or both versus neither of these signs. All archived pancreata samples were evaluated histologically and scored using a published pancreatic scoring system.
\end{abstract}

A total of 61 dogs with chronic pancreatitis were included in the study. The most frequent clinical signs were lethargy, decreased appetite, vomiting, and diarrhea. Compared to the reference necropsy population, chronic pancreatitis cases were more likely to be older, neutered, 
and of the non-sporting/toy breed group and to have concurrent endocrine, hepatobiliary, or neurologic diseases. Clinical chronic pancreatitis cases had significantly higher histological scores for pancreatic necrosis and peripancreatic fat necrosis. Clinical chronic pancreatitis cases were significantly more likely to have hepatobiliary or endocrine disease as well as increased liver enzyme activities, and cholesterol and bilirubin concentrations. In conclusion, clinical disease resulting from chronic pancreatitis might be related to the presence of pancreatic necrosis and pancreatic fat necrosis. The signalment, presentation, and concurrent diseases of dogs with chronic pancreatitis are similar to those previously reported for dogs with acute pancreatitis.

Keywords: Canine; Chronic pancreatitis; Pancreatic inflammation; Histopathology: Exocrine pancreatic disease.

\section{Introduction}

Chronic pancreatitis has not been sufficiently characterized in dogs. The epidemiology, pathophysiology, clinical presentation, diagnosis, clinical importance, and treatment of chronic pancreatitis in dogs are all aspects of the disease that have been insufficiently studied and poorly defined (Xenoulis et al., 2008).

Published information on the clinical, clinicopathological, and histopathologic findings of dogs with chronic pancreatitis, as well as any associations among them, is very limited. Two recently published studies described the clinical and clinicopathological findings of a small number of dogs with histologically confirmed chronic pancreatitis, but these studies were both conducted in the UK and included mainly English Cocker Spaniels and Cavalier King Charles 
Spaniels, which were suggested by the authors to have a distinct form of chronic pancreatitis (Watson et al., 2010a; Watson et al., 2011).

Recent published information on canine chronic pancreatitis is almost exclusively based on histopathology studies (Newman et al., 2004; Watson et al., 2007). In those studies, histopathologic findings suggestive of chronic pancreatitis were present in an unexpectedly large number of dogs, ranging between $34 \%$ and $64 \%$ of all cases examined (Newman et al., 2004; Watson et al., 2007). However, these studies do not provide any information on how these histopahological findings translate into clinical disease; therefore, the clinical importance of those findings remains unknown. Although clinical studies on the incidence on canine chronic pancreatitis are lacking, clinical experience suggests that this condition is diagnosed much less frequently in clinical practice, which might suggest that many chronic pancreatitis cases are missed or remain subclinical (Xenoulis et al., 2008).

Due to the difficulties in the clinical diagnosis of chronic pancreatitis, histopathology has been considered to be the most reliable means of diagnosis of this condition in dogs (Xenoulis et al., 2008). However, unlike humans, a consensus regarding the terminology, classification, and differentiation of different histopathologic forms of pancreatitis is currently not available for dogs. Therefore, reported studies on chronic pancreatitis in dogs often use different definitions and grading schemes of chronic pancreatitis in this species (Xenoulis et al., 2008).

Clinical studies investigating chronic pancreatitis in dogs are important in order to better define and understand the clinical aspects of this condition. Similarly, elucidation of the 
association between clinical presentation and histopathological findings of dogs with chronic pancreatitis is of major clinical importance. The purpose of this study was to retrospectively describe the clinical, clinicopathological, and histopathological findings in dogs with chronic pancreatitis identified during necropsy examination.

\section{Materials and methods}

\section{Case selection}

The Texas A\&M University Veterinary Teaching Hospital (TAMU-VTH) necropsy database was searched electronically for cases between the years of 1992 to 2008 using the keywords 'chronic pancreatitis'. Necropsy reports were retrieved and reviewed to confirm a diagnosis of chronic pancreatitis. For the purpose of this study, chronic pancreatitis was defined solely based on histopathologic criteria, as previously described (Newman et al., 2006). These criteria included inflammatory (neutrophilic and/or lymphocytic) infiltration of the pancreas and irreversible changes, pancreatic atrophy and fibrosis. Necropsy cases that were not admitted through TAMU-VTH, had incomplete necropsy reports, had predominately histiocytic inflammation, or had visible infectious organisms or neoplasia within the pancreas, were excluded.

\section{Review of medical records and data description}

All available medical records for the selected cases were reviewed and data regarding clinical presentation, complete blood cell count, serum biochemical analysis, ultrasonography, concurrent diseases, length of hospitalization (if the dogs were hospitalized), medications, and additional necropsy report details were extracted and tabulated. For the purpose of this study, 
clinical signs were designated acute in nature if present for less than 3 weeks and chronic if present for greater than 3 weeks. If multiple sets of bloodwork were available, only the initial set of results from the most recent admission was used for analysis. Only medications given during hospitalization and the 3 weeks previous to hospitalization were recorded. Medications given were classified as an antibiotic, antiepileptic, corticosteroid, plasma, or other.

\section{Clinical classification of cases}

Cases that fulfilled the inclusion criteria for chronic pancreatitis mentioned above were categorized into one of the following 2 groups based on clinical presentation: clinical chronic pancreatitis or incidental chronic pancreatitis. Based on the small number of cases that have been reported in the literature (Watson et al., 2010a; Watson et al., 2011) and the authors' clinical experience, the most consistent clinical signs of dogs with chronic pancreatitis are chronic or intermittent vomiting and decreased appetite. Therefore, dogs were classified as having clinical chronic pancreatitis if they had presenting clinical signs of vomiting and/or decreased appetite. Dogs were classified as having incidental chronic pancreatitis if none of these signs were present.

\section{Concurrent diseases}

Concurrent diseases of dogs with chronic pancreatitis were recorded and categorized by body system or process as follows: endocrine, autoimmune, neoplastic, neurologic, gastrointestinal, hepatobiliary, respiratory, urologic, cardiac, infectious, and trauma. 


\section{Histological grading}

Archived hematoxylin and eosin stained slides of the pancreas were reviewed and graded by a single board-certified pathologist ( $\mathrm{SN}$ ) based on a published grading scheme (Newman et al., 2006). Each slide was assessed for the following: neutrophilic inflammation, lymphocytic inflammation, pancreatic necrosis, pancreatic fat necrosis, edema, fibrosis, atrophy, and hyperplastic nodules. Each parameter was subsequently graded; with grade 0 representing the absence of said parameter, grade 1 representing that $<10 \%$ of the section had been affected, grade 2 representing that $10-40 \%$ of the section had been affected, and grade 3 representing that $>40 \%$ of the section had been affected. If more than one slide was present for evaluation, a mean cumulative severity score was assigned (Newman et al., 2006).

\section{Reference population selection}

In order to more accurately illustrate possible associations of histological lesions of chronic pancreatitis with signalment (i.e., age, sex, weight, and breed) and concurrent diseases, a reference population of dogs was established from the same necropsy database. The reference population consisted of 100 randomly selected dogs (using a random number generator) submitted for routine necropsy during the same time period (1992-2008). Dogs that were not admitted through TAMU-VTH or had incomplete necropsy reports were excluded. None of the dogs in the reference population had pancreatic disease listed as one of their final diagnoses. Data regarding signalment and final diagnosis were extracted from necropsy reports. These data were used for comparisons between the cases and the reference population only with regards to signalment and concurrent diseases; detailed data for the reference population are not shown due to space limitations. 


\section{Statistical analyses}

Descriptive statistics including medians and ranges were calculated for quantitative variables including age, weight, complete blood count values, and biochemistry parameters. Categorical variables, including breed, sex, treatments, concurrent diseases, and presenting clinical signs were summarized by counts, percentages, and mid- $P$ exact $95 \%$ confidence intervals (CI). Due to the small number of cases for each individual breed, cases were assigned to a breed group using American Kennel Club (AKC) breed groupings (American Kennel Club 2006). Quantitative variables were assessed for the assumption of normality through the visual inspection of histograms, descriptive statistics, and by using the Anderson-Darling test for normality. Comparisons were made between the pancreatitis cases and the reference population regarding signalment and concurrent diseases (or final diagnoses in the reference population) using Mann-Whitney U tests for quantitative variables and Pearson chi-square or Fisher's exact tests for categorical variables. Comparisons were also made between incidental and clinical chronic pancreatitis cases regarding signalment, clinical signs, clinical pathology data, histologic grading, and concurrent diseases using Mann-Whitney, Pearson chi-square, or Fisher's exact tests. Categorical variables were further summarized by calculating odds ratios (OR) and their corresponding 95\% CI. Statistical analysis was performed using standard computer packages

(Epi Info 6.04, Centers for Disease Control; SPSS 15.0 for Windows; MINITAB Statistical Software 13.32, Minitab Inc.,) and the significance set at a $P \leq 0.05$ for all testing. 


\section{Results}

\section{Cases and clinical classification}

The initial search for 'chronic pancreatitis' returned 150 necropsy cases. From those a total of 61 dogs with chronic pancreatitis were included in the study after final review. Of the 61 dogs, $40(66 \%)$ dogs were classified as having clinical chronic pancreatitis and 18 (29\%) were classified as having incidental chronic pancreatitis cases; three dogs (5\%) could not be definitively classified due to insufficient and ambiguous medical records and were subsequently excluded from comparisons between clinical and incidental cases. However, they were included in all other analyses and comparisons with the reference group because they fulfilled the histopathologic criteria for chronic pancreatitis.

\section{Signalment}

Thirty-three breeds were represented in the group of dogs with chronic pancreatitis. Mixed-breed dogs $(5 / 61 ; 8 \%)$ and Labrador retrievers $(5 / 61 ; 8 \%)$ were the most common dog breeds represented. Other breeds commonly represented were Boxers $(4 / 61 ; 7 \%)$, Miniature Schnauzers $(4 / 61 ; 7 \%)$, Collies $(3 / 61 ; 5 \%)$, Dachshunds $(3 / 61 ; 5 \%)$, Dalmatians $(3 / 61 ; 5 \%)$, and Golden retrievers $(3 / 61 ; 5 \%)$. Compared to the reference population, dogs with chronic pancreatitis were significantly more likely to be pure-bred (OR 3.35, 95\% CI 1.14-11.9, $P=$ 0.016) and more specifically belonging to the $\mathrm{AKC}$ toy and non-sporting breeds combined (OR 2.83, 95\% CI 1.16-6.99, $P=0.011)$. Also, AKC toy and non-sporting breeds combined had a significantly higher likelihood of being categorized as clinical chronic pancreatitis as opposed to incidental chronic pancreatitis (OR 10.2, 95\% CI 1.28-454, $P=0.012$ ). 
The median age of the case dogs included in this study was 10 years (range: 1 to 16 years) which was significantly older than the necropsy reference population (median age: 6, range 1 month to 18 years, $P<0.001)$. There was no statistically significant difference $(P=0.29)$ between the ages of the clinical and incidental pancreatitis cases.

Thirty-nine case dogs (64\%) were female (4 sexually intact, 35 spayed) and $22(36 \%)$ were male (4 sexually intact, 18 castrated). Compared to the reference necropsy population, chronic pancreatitis patients were more likely to be neutered regardless of gender (OR 4.64, 95\% CI $1.80-12.5, P<0.001)$ and female (OR 2.17, 95\% CI 1.07-4.40, $P=0.020$ ). There was no significant difference in sex or sexual status between the groups of dogs with clinical or incidental chronic pancreatitis.

The median weight of the dogs with chronic pancreatitis was $19.3 \mathrm{~kg}$ (range: $1.1-45.8$ $\mathrm{kg})$. Weight did not differ significantly between the reference and study population $(P=0.493)$ or between the clinical and incidental pancreatitis cases $(P=0.051)$.

\section{Clinical signs and physical examination findings}

The most common clinical sign in the chronic pancreatitis group was lethargy $(45 / 56$; $80 \%)$, followed by a decreased appetite $(39 / 56 ; 70 \%)$, vomiting $(35 / 56 ; 63 \%)$, and diarrhea $(20 / 55 ; 36 \%)$. Eighteen of 52 dogs $(35 \%)$ had a history of chronic vomiting that was defined as vomiting episodes occurring for more than 3 weeks prior to presentation. Fourteen of 52 dogs (27\%) had evidence of abdominal pain on physical examination, while 12 of 53 dogs (23\%) had an elevated body temperature $\left(>39.7^{\circ} \mathrm{C}\right)$ on presentation. 
Table 1. Select clinical signs, physical examination findings, concurrent diseases, medications administered, and necropsy findings of dogs with clinical $(n=40)$ or incidental chronic pancreatitis $(n=18)$. By definition, incidental cases did not show vomiting or decreased appetite.

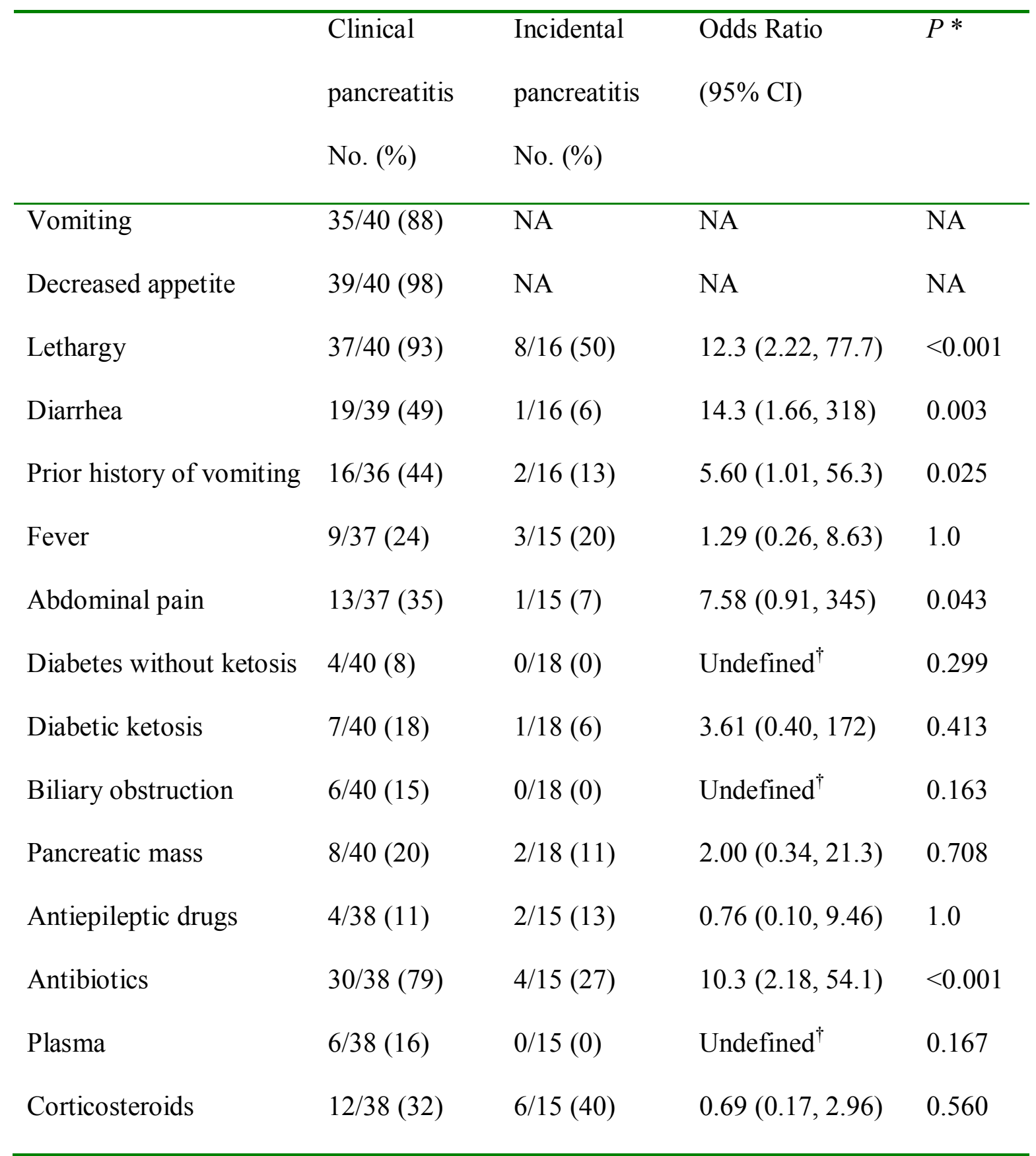

$\mathrm{NA}=$ not applicable.

*Comparison of clinical and incidental proportions using Pearson chi-square or Fisher exact tests

${ }^{\dagger}$ Odds ratio and confidence interval are undefined because the denominator is zero 
Clinical chronic pancreatitis cases were significantly more likely than incidental cases to have diarrhea, lethargy, abdominal pain, and a history of chronic vomiting (Table 1). Decreased appetite and vomiting was inherent to our definition of clinical pancreatitis and could not be evaluated.

\section{Medications}

Antiepileptic medications that were reported to have been used included phenobarbital, phenytoin, primidone, and potassium bromide. Two patients received azathioprine (one from each of the clinical and incidental groups). The only significant difference between the clinical and incidental chronic pancreatitis cases with respect to medications was that dogs with clinical chronic pancreatitis were more likely to have received antibiotics (Table 1).

\section{Concurrent diseases}

Chronic pancreatitis dogs were significantly more likely to have concurrent endocrine diseases $(18 / 61$; OR $13.5,95 \%$ CI $3.50-61.3, P<0.001)$ than the reference necropsy cases (3/100). Specifically, of the 61 dogs with chronic pancreatitis, 12 had diabetes mellitus, either with $(n=8)$ or without $(n=4)$ ketosis, 4 had hypothyroidism, 1 had hyperadrenocorticism, and 1 had hypoadrenocorticism. In the reference population, 2/100 dogs had hyperadrenocorticism and 1 dog had post partum eclampsia. Dogs with chronic pancreatitis were significantly more likely to have diabetes mellitus (OR and 95\% CI undefined due to the denominator being zero, $P<$ 0.0001 ) or hypothyroidism (OR and $95 \% \mathrm{CI}$ undefined due to the denominator being zero, $P=$ 0.02) compared to the reference population. In addition, chronic pancreatitis dogs were significantly more likely to have concurrent neurologic (14/61; OR 2.68, 95\% CI 1.02-7.11, $P=$ 
$0.025)$ or hepatobiliary disease $(11 / 61$; OR $3.45,95 \%$ CI $1.09-11.2, P=0.016)$, and significantly less likely to have infectious diseases $(0 / 61$; OR $0,95 \%$ CI $0-0.69, P=0.014)$ or trauma $(0 / 61$;

OR $0,95 \%$ CI $0-0.55, P=0.004)$ than the reference necropsy cases $(10 / 100,6 / 100,10 / 100$, and $12 / 100$, respectively).

Dogs with clinical chronic pancreatitis were significantly more likely to have concurrent hepatobiliary disease than dogs with incidental chronic pancreatitis (11/40 versus $0 / 18$, respectively; OR and $95 \% \mathrm{CI}$ undefined due to the denominator being zero, $P=0.012$ ). In contrast, dogs with incidental chronic pancreatitis were more likely to have concurrent neoplasia (9/18 vesrus 6/40; OR 5.67, 95\% CI 1.36-24.8, $P=0.009)$.

\section{Clinicopathological findings}

The clinicopathological findings of dogs with chronic pancreatitis that had serum biochemical and hematologic analyses performed are summarized in Tables 2 and 3, respectively. Clinical chronic pancreatitis cases had significantly higher serum bilirubin and cholesterol concentrations and alanine aminotransferase, alkaline phosphatase, and gammaglutamyltransferase activities than those with incidental chronic pancreatitis (Table 4).

Serum cPLI concentrations were measured in 9 dogs classified as having clinical pancreatitis and 6 of those dogs $(67 \%)$ had serum concentrations greater than the upper limit of the reference range $(>102.1 \mu \mathrm{g} / \mathrm{L}$ as measured with the in-house ELISA, $>200 \mu \mathrm{g} / \mathrm{L}$ as measured by Spec cPL); the same 6 dogs also had serum cPLI concentrations that were above the cut-off 
Table 2. Results of serum biochemical analysis in 47 of 61 dogs with histopathologic evidence of chronic pancreatitis included in this retrospective case series.

\begin{tabular}{|c|c|c|c|c|}
\hline Variable & RI & $\begin{array}{l}\text { No. }(\%) \\
\text { dogs }>\text { RI }\end{array}$ & $\begin{array}{l}\text { No. }(\%) \\
\operatorname{dog}_{\mathrm{S}}<\mathrm{RI}\end{array}$ & Median (range) \\
\hline Cholesterol (mg/dL) & $120-247$ & $23(49)$ & $6(13)$ & $234(0-852)$ \\
\hline $\operatorname{ALP}(\mathrm{U} / \mathrm{L})$ & $24-147$ & $39(83)$ & $2(4)$ & $794.5(20-7667)$ \\
\hline $\operatorname{ALT}(\mathrm{U} / \mathrm{L})$ & $10-130$ & $22(47)$ & $0(0)$ & $106(11-2835)$ \\
\hline GGT (U/L) & $0-25$ & $21(45)$ & NA & $25(0-320)$ \\
\hline Bilirubin (mg/dL) & $0-0.8$ & $20(43)$ & NA & $0.6(0-19.6)$ \\
\hline Albumin $(\mathrm{g} / \mathrm{dL})$ & $2.4-3.6$ & $18(38)$ & $1(2)$ & $2.6(1.4-3.9)$ \\
\hline Globulin (g/dL) & $1.7-3.8$ & $13(28)$ & $3(6)$ & $3.2(0.7-5.4)$ \\
\hline Total protein $(\mathrm{g} / \mathrm{dL})$ & $5.7-7.8$ & $4(8)$ & $13(28)$ & $5.7(2.3-8.5)$ \\
\hline Potassium (mmol/L) & $3.3-4.6$ & $7(15)$ & $10(21)$ & $3.8(2-6.6)$ \\
\hline Chloride ( $\mathrm{mmol} / \mathrm{L})$ & $107-116$ & $15(32)$ & $15(32)$ & $111(83-144)$ \\
\hline Calcium (mmol/L) & $9.3-11.8$ & $5(11)$ & $19(40)$ & $9.4(2.1-13.8)$ \\
\hline $\mathrm{ECO}_{2}(\mathrm{mmol} / \mathrm{L})$ & $21-28$ & $2(4)$ & $24(51)$ & $20(8-32)$ \\
\hline Glucose (mg/dL) & $60-135$ & $14(30)$ & $1(2)$ & $112(57-875)$ \\
\hline BUN (mg/dL) & $5-29$ & $16(34)$ & $1(2)$ & $19(3-224)$ \\
\hline Creatinine $(\mathrm{mg} / \mathrm{dL})$ & $0.3-2.0$ & $10(21)$ & $0(0)$ & $1(0.3-12.1)$ \\
\hline Phosphorus (mg/dL) & $2.9-6.2$ & $9(19)$ & $3(6)$ & $5(1.4-29.9)$ \\
\hline
\end{tabular}

ALP $=$ alkaline phosphatase. ALT $=$ Alanine aminotransferase. GGT = gammaglutamyltransferase. $\mathrm{ECO}_{2}=$ enzymatically measured carbon dioxide. $\mathrm{BUN}=$ blood urea nitrogen. $\mathrm{NA}=$ not applicable. $\mathrm{RI}=$ reference interval. 
Table 3. Results of hematologic analysis in 47 of 61 dogs with histopathologic evidence of chronic pancreatitis included in this retrospective case series.

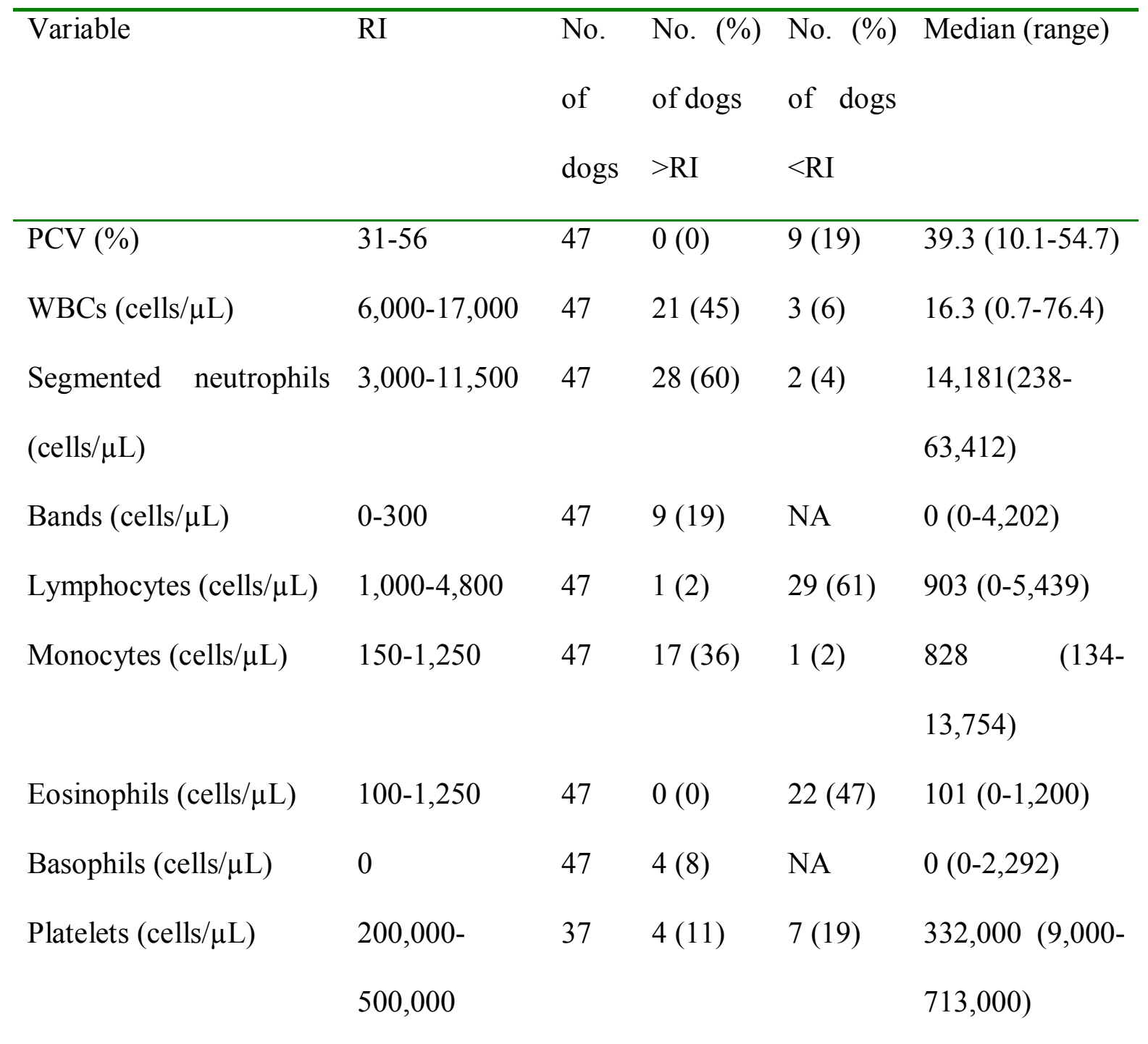

$\mathrm{PCV}=$ Packed cell volume. $\mathrm{WBC}=$ white blood cells. $\mathrm{RI}=$ reference interval. $\mathrm{NA}=$ not applicable 
Table 4. Results of select variables from serum biochemical analysis in dogs with clinical or incidental chronic pancreatitis.

\begin{tabular}{|c|c|c|c|c|c|c|}
\hline Variable & RI & $\begin{array}{l}\text { No. of } \\
\text { dogs }\end{array}$ & $\begin{array}{l}\text { No. }(\%) \text { of } \\
\text { dogs }>\text { RI }\end{array}$ & $\begin{array}{l}\text { No. }(\%) \text { of } \\
\text { dogs }<\text { RI }\end{array}$ & Median (range) & $P^{*}$ \\
\hline Cholesterol & $120-247$ & & & & & \\
\hline Clinical & $\mathrm{mg} / \mathrm{dL}$ & 35 & $19(54)$ & $3(9)$ & $262.5(0-852)$ & 0.032 \\
\hline Incidental & & 10 & $2(20)$ & $1(10)$ & $186(81-281)$ & \\
\hline ALP & $24-147$ & & & & & \\
\hline Clinical & $\mathrm{U} / \mathrm{L}$ & 35 & $30(85)$ & $2(6)$ & $1103(21-7667)$ & 0.016 \\
\hline Incidental & & 10 & $7(70)$ & $1(10)$ & $246.5(20-2013)$ & \\
\hline ALT & $10-130$ & & & & & \\
\hline Clinical & $\mathrm{U} / \mathrm{L}$ & 35 & $20(57)$ & $0(0)$ & $187(21-2835)$ & 0.006 \\
\hline Incidental & & 10 & $2(20)$ & $0(0)$ & $38(11-224)$ & \\
\hline GGT & $0-25$ & & & & & \\
\hline Clinical & $\mathrm{U} / \mathrm{L}$ & 35 & $18(51)$ & NA & $27(0-320)$ & 0.008 \\
\hline Incidental & & 10 & $2(20)$ & NA & $8(5-30)$ & \\
\hline Bilirubin & $0-0.8$ & & & & & \\
\hline Clinical & $\mathrm{mg} / \mathrm{dl}$ & 35 & $17(49)$ & NA & $0.9(0-19.6)$ & 0.018 \\
\hline Incidental & & 10 & $0(0)$ & NA & $0.3(0.1-1.3)$ & \\
\hline
\end{tabular}

$\mathrm{ALP}=$ alkaline phosphatase. $\mathrm{ALT}=$ Alanine aminotransferase. GGT = gammaglutamyltransferase. $\mathrm{RI}=$ reference interval. $\mathrm{NA}=$ not applicable.

*Based on the comparison of clinical and incidental values using Mann-Whitney U tests. 
value $(>200 \mu \mathrm{g} / \mathrm{L}$ as measured with the in-house ELISA, $>400 \mu \mathrm{g} / \mathrm{L}$ as measured by Spec cPL) for a diagnosis of pancreatitis.

Histologic grading - The results of the histologic grading of the 61 hematoxylin and eosin stained pancreata are summarized as follows: fibrosis (median: 1, range: $0-3$ ), pancreatic necrosis (median: 0, range: 0-3), peripancreatic fat necrosis (median: 0, range: 0-3), edema (median: 0, range: 0-1.5), neutrophilic inflammation (median: 0, range: 0-3), lymphocytic inflammation (median: 1, range: 0-2), atrophy (median: 0.33, range: 0-3), and hyperplastic nodules (median: 1 , range: $0-3$ ).

Clinical cases had significantly more peripancreatic fat necrosis $(P=0.010)$ and pancreatic necrosis $(P=0.015)$ than incidental cases (Figs. 1 and 2).

\section{Additional clinical findings}

Dogs with clinical chronic pancreatitis were hospitalized for a significantly greater number of days (mean: 2 days, range 0 to 34 days, $P=0.031$ ) than dogs with incidental chronic pancreatitis (mean 0 days, range 0 to 6 days).

Thirty-seven of the 61 dogs $(60 \%)$ had an abdominal ultrasound examination performed. For $12 / 37$ dogs $(32 \%)$ who had an abdominal ultrasound the pancreas was not mentioned in the ultrasound report; for $5 / 37$ dogs $(16 \%)$ the pancreas was reported to be normal; for $19 / 37$ dogs (51\%) the pancreas was reported to be abnormal; for $1 / 37$ dogs (3\%) pain was noted in the area 


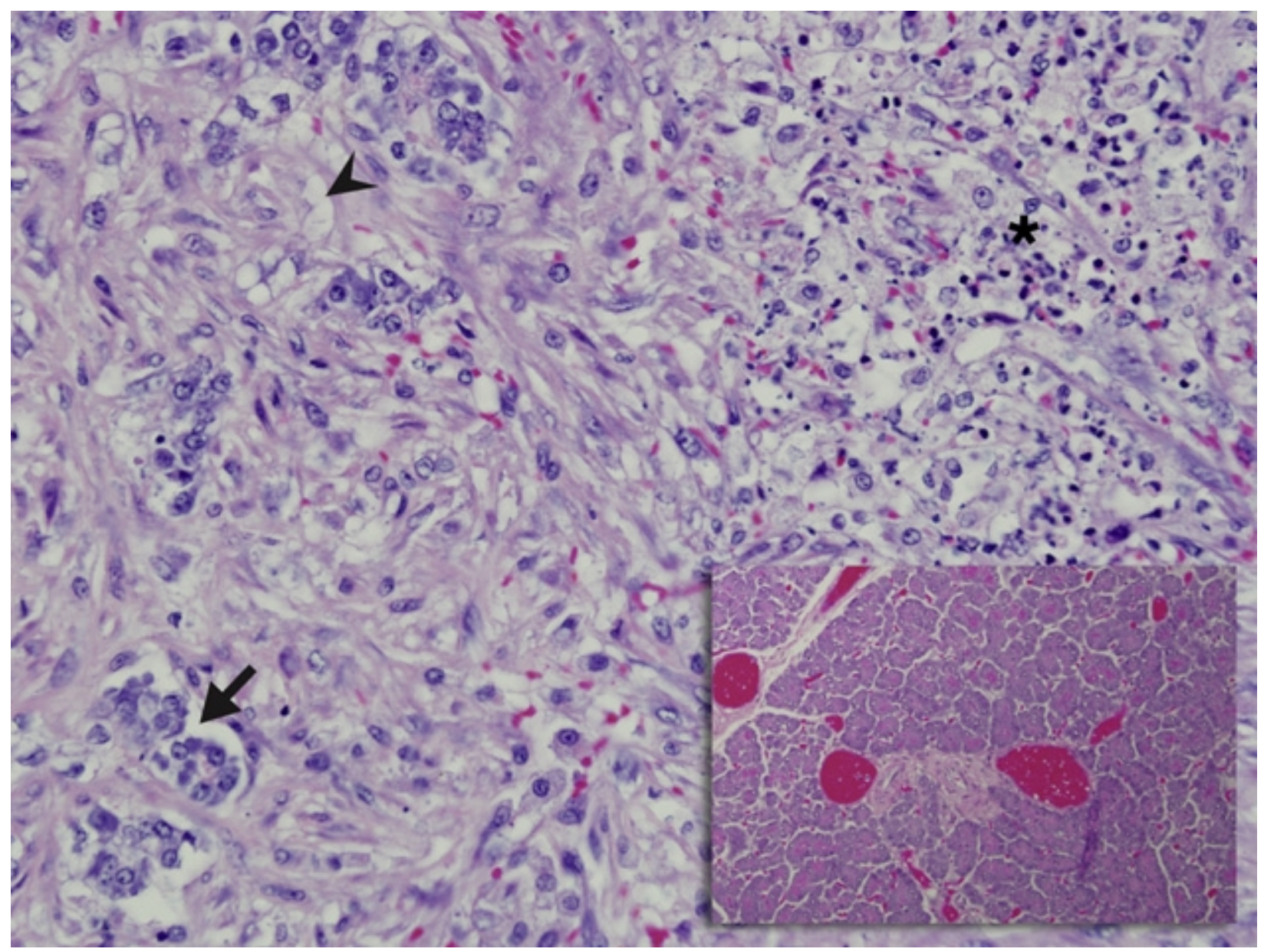

Figure 1: Pancreas from a dog in the group of clinical pancreatitis. Focal necrosis and infiltration by degenerative neutrophils is present (asterix). Pancreatic acini are separated by abundant mature fibrous connective tissue (arrowhead) and there is loss of pancreatic parenchyma (atrophy) [arrow]. Hematoxlyin and eosin (20X). Inset: Normal pancreas for comparison (10X). 


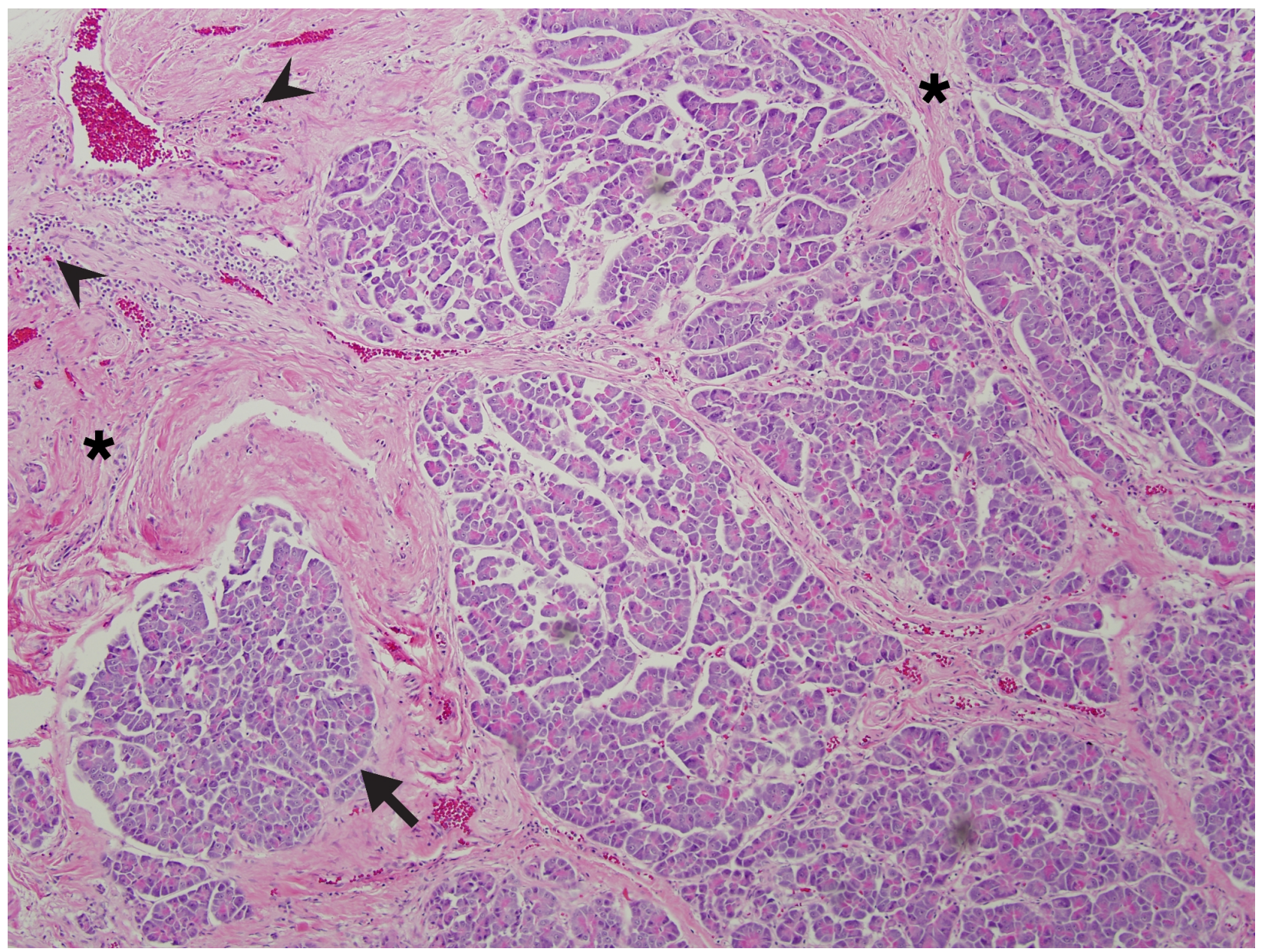

Figure 2: Pancreas from a dog in the group of incidental pancreatitis. Inflammation in minimal and seen as a few lymphocytic infiltrates (arrowheads). Pancreatic tissue is arranged in proliferative lobules (arrow) and separated by abundant mature fibrous connective tissue (fibrosis) [asterix]. Hematoxlyin and eosin (10X).

of the pancreas but the ultrasonographer was unable to visualize the pancreas. Eighty-three percent of dogs (19/23) with clinical chronic pancreatitis had an abnormal pancreas compared to none $(0 / 1)$ in the incidental group $(P=0.208)$. 


\section{Discussion}

To the authors' knowledge, this is the largest study evaluating the clinical, clinicopathologic, and histopathologic findings in a series of dogs of various breeds with chronic pancreatitis. One of the most important findings of the present study is that dogs with clinical chronic pancreatitis had significantly higher histological scores for pancreatic necrosis and peripancreatic fat necrosis than dogs with incidental chronic pancreatitis. These two histopathologic findings are typically associated with acute pancreatitis (Newman et al., 2006; Xenoulis et al., 2008), and may indicate that many dogs in the group of clinical chronic pancreatitis also had acute pancreatitis. The presence of active pancreatic disease (as indicated by pancreatic and peripancreatic fat necrosis) in the clinical chronic pancreatitis dogs likely accounts for the higher prevalence of clinical signs in this group of dogs compared to that of the incidental chronic pancreatitis group. This might provide a plausible explanation for the discrepancy of the prevalence of chronic pancreatitis between necropsy studies and clinical impressions (Newman et al., 2006; Watson et al., 2007; Xenoulis et al., 2008; Watson et al., 2010a). Chronic pancreatitis cases that are not associated with pancreatic and peripancreatic fat necrosis are less likely to develop clinical signs, and therefore, they might escape clinical diagnosis more easily. However, these cases are identified as chronic pancreatitis cases based on histopathology.

The finding of the presence of pancreatic and peripancreatic fat necrosis in this study might also support the necrosis-fibrosis theory as a possible explanation for the pathogenesis of chronic pancreatitis in dogs. The necrosis-fibrosis theory of chronic pancreatitis explains that the irreversible damage to the pancreas may be caused by repeated incidents of acute pancreatitis 
(Witt et al., 2007). This theory is also supported by the similarity of the signalment and clinical signs reported in dogs with acute pancreatitis and those found in this study of dogs with chronic pancreatitis (Cook et al., 1993; Hess et al., 1998).

This study supports findings of previous smaller studies and clinical impressions that chronic pancreatitis in dogs is associated with vague clinical signs and indistinct clinicopathological findings (Xenoulis et al., 2008; Watson et al., 2010a; Watson et al., 2011). The most common clinical signs identified in this study were lethargy, decreased appetite, vomiting, and diarrhea. The presence of clinical signs such as diarrhea, lethargy, abdominal pain, and a history of chronic vomiting were seen significantly more common in dogs with clinical disease than dogs with incidental disease, indicating that a more severe clinical presentation is associated with a more active pancreatic pathology (as indicated by pancreatic and peripancreatic fat necrosis). Given that the clinical presentation of dogs with acute pancreatitis (Hess et al., 1998) is very similar to the one reported here for dogs with clinical chronic pancreatitis, it is quite possible that some cases diagnosed clinically as having acute pancreatitis might in fact have an acute exacerbation of chronic disease. This might provide an additional plausible explanation of the discrepancy of the prevalence of chronic pancreatitis between necropsy studies and clinical impressions (Newman et al., 2006; Watson et al., 2007; Xenoulis et al., 2008; Watson et al., 2010a). The most common clinicopathologic abnormalities observed were increased serum liver enzyme activities, and serum bilirubin, and cholesterol concentrations; these findings agreed with other studies in dogs with chronic (Watson et al., 2010a) and acute pancreatitis (Hess et al., 1998). 
Breed predispositions in dogs with histopathological evidence of chronic pancreatitis in the present study showed similarities with those reported for dogs with acute pancreatitis (Cook et al., 1993; Hess et al., 1998; Lem et al., 2008). Previous studies found that dogs of the nonsporting and terrier breed groups had an increased likelihood for acute pancreatitis (Cook et al., 1993; Hess et al., 1998; Lem et al., 2008). Although a significant overrepresentation of the terrier group was not evident in the present study, we found that non-sporting and toy breeds were at a higher risk for chronic pancreatitis than other breed groups. In contrast, studies on canine chronic pancreatitis from the UK have suggested an increased relative risk for pancreatitis in Cavalier King Charles Spaniels and Cocker Spaniels; however, neither breed was identified to be overrepresented in the present study. This might suggest that breed-related predispositions for pancreatitis might differ among geographic regions depending on the breeding lines used for reproduction. Finally, in accordance to previous studies, older neutered dogs in this study were more likely to have chronic pancreatitis compared to the necropsy reference population (Cook et al., 1993; Hess et al., 1998; Lem et al., 2008). The similarity in the signalment between dogs with acute and chronic pancreatitis might provide further supportive evidence of the theory that chronic pancreatitis might be the result of repeated bouts of acute pancreatitis in dogs.

Dogs with chronic pancreatitis in the present study were more likely to have post-mortem evidence of hepatobiliary disease than were dogs of the reference necropsy population. An association between pancreatitis (mainly acute) and hepatobiliary disease has been described in several studies, although the exact relationship between the two conditions is not always clearly defined (Hess et al., 1998; Hess et al., 1999). The exact nature of hepatic disease was not described in the present study, but a recent study reported that dogs with chronic pancreatitis 
were at increased risk for having histopathological evidence of reactive hepatopathy (Watson et al., 2010b). In addition, swelling from pancreatic inflammation and edema in addition to scarring and fibrosis can lead to functional obstruction of the common bile duct because of its anatomically intimate association with the pancreas (Mehler et al., 2006). In the present study, 6 dogs in the clinical chronic pancreatitis group were diagnosed as having biliary obstruction.

In the present study, dogs with clinical chronic pancreatitis were more likely to have both clinicopathological (i.e., increased serum liver enzyme activities and bilirubin concentration) and histopathological evidence of concurrent hepatobiliary disease when compared with dogs with incidental chronic pancreatitis. This might be associated with the fact that dogs with clinical chronic pancreatitis have more histopathologically active pancreatic disease as indicated by the presence of pancreatic and peripancreatic fat necrosis. In this study it was difficult to assess if dogs with an increased serum bilirubin concentration had any definitive evidence of extrahepatic biliary obstruction retrospectively because many dogs had confirmed patent bile ducts or in other dogs the patency was not assessed during necropsy. In addition, it was not possible to determine the cause-and-effect relationship between pancreatitis and hepatobiliary disease.

Chronic pancreatitis cases were significantly more likely to have an endocrine disease, specifically diabetes mellitus or hypothyroidism, than the reference necropsy cases. Such a relationship between pancreatitis and certain endocrinopathies has previously been described for both acute and chronic pancreatitis, although this relationship was poorly defined (Hess et al., 1998; Hess et al., 1999; Xenoulis et al., 2008; Causmaecker et al., 2009). There has been much debate over cause and effect, e.g., whether those endocrine diseases can directly predispose to 
pancreatitis, whether hyperlipidemia associated with those endocrine disorders may play a role, or whether pancreatitis predisposes to the development of certain endocrine diseases (e.g., diabetes mellitus) (Xenoulis and Steiner 2010). The fact that in this study hypercholesterolemia was significantly more common in dogs with chronic pancreatitis might be related to the fact that these dogs more commonly had endocrine diseases, many of which (e.g., diabetes mellitus, hyperadrenocorticism, hypothyroidism) are known to increase serum cholesterol concentrations (Xenoulis and Steiner 2010). The clinical chronic pancreatitis cases were not more likely to have an endocrine disease than the incidental ones.

In this study, dogs with chronic pancreatitis were more likely to have concurrent neurologic disease compared to the necropsy reference population. This is in agreement with the findings of an older study (Hess et al., 1999). One possible explanation for this finding is that anti-epileptic medications, specifically potassium bromide and phenobarbital, have been associated with increased serum cPLI concentrations in dogs, thus suggesting an increased risk for pancreatitis (Steiner et al., 2008). However, in this group of dogs, the clinical chronic pancreatitis cases were not more likely to have been administered anti-epileptic drugs compared to the incidental pancreatitis cases. Another possible explanation for this finding is that certain antiepileptic medications (phenobarbital and potassium bromide) have been associated with hypertriglyceridemia (Kluger et al., 2008), which in dogs is considered a risk factor for pancreatitis (Xenoulis and Steiner, 2010; Xenoulis et al, 2010; Xenoulis et al., 2011). Unfortunately, serum triglyceride concentrations were not available for dogs in this study. 
In contrast to the findings of a recent study from the UK, dogs with chronic pancreatitis in this study were not more likely to have exocrine pancreatic insufficiency (EPI) than the control population (Watson 2010a). A plausible explanation for this finding might be that in the study from the UK dogs were initially enrolled based on the presence of compatible clinical signs, which might have led to the exclusion of dogs with very mild or subclinical chronic pancreatitis, leading to an overestimation of the prevalence of EPI among dogs with chronic pancreatitis. In contrast, in the present study, dogs were selected based on histopathologic findings and mild cases were not excluded. Alternatively, EPI as a result of chronic pancreatitis might be more common in specific dog breeds represented more commonly in the UK study (Watson 2010a), such as English Cocker Spaniels and Cavalier King Charles Spaniels.

The interpretation of the ultrasound findings needs to be done with caution. For a large number of dogs, the pancreas was not mentioned in the report. This might mean that it was not examined or that it was normal. Due to the retrospective nature of this study, it is not possible to know which one is true for each case and this can dramatically affect the interpretation of the results. Similarly, serum cPLI concentrations were available for only 9 dogs. Although the sensitivity was relatively high (almost 70\%), it is very likely that the case selection of cases for measurement of serum cPLI was subject to clinician bias. Therefore, additional studies need to be performed in order to more accurately investigate the performance of ultrasonography and serum cPLI in the diagnosis of canine chronic pancreatitis.

Given the retrospective nature of this study there are several inherent limitations, such as incomplete medical records, non-standardized diagnostics, and clinician bias. Another limitation 
was that the impact of hypertriglyceridemia on chronic pancreatitis (Xenoulis and Steiner, 2010; Xenoulis et al., 2010; Xenoulis et al., 2011) was not assessed due to the fact that the routine chemistry profile at the Small Animal Teaching Hospital at Texas A\&M University does not include measurement of serum triglyceride concentration. Also, due to the large number of concurrent diseases, it is difficult to confidently tie the reported clinical signs specifically to chronic pancreatitis and not to one or more of the concurrent diseases. In addition, due the vague and poorly defined clinical presentation of dogs with chronic pancreatitis, the selection of clinical signs that define the clinical picture of the disease is hard. Another important limitation is the fact that these cases were collected from a necropsy population. During necropsy at TAMU VTH the pancreas is not routinely sampled for histopathologic examination, nor is it sampled in multiple locations when a sample is being collected. Inflammatory lesions of the pancreas are often subtle during gross examination and can be sporadic and multifocal microscopically, making them difficult to identify in those cases when only one sample is collected (Newman et al., 2004). Because of these limitations, additional studies comparing histological and clinicopathological findings between acute and chronic pancreatitis are warranted.

\section{Conclusions}

The present study provided for the first time evidence that clinical signs associated with chronic pancreatitis are more likely to occur in association with a histopathologically more active disease as determined by the presence of pancreatic and peripancreatic fat necrosis. In addition, this study provided further evidence that chronic pancreatitis may be an incidental finding in some cases, while in other cases it is associated with non-specific clinical and clinicopathological findings. Signalment and clinical presentation of dogs with chronic pancreatitis was very similar 
to those reported in previous studies for dogs with acute pancreatitis. Concurrent diseases, mainly hepatobiliary and endocrine disease, are common in dogs with chronic pancreatitis, although the exact relationship between those diseases and pancreatitis warrants further investigation, ideally using a cohort study design.

\section{Conflict of interest}

None of the authors of this paper has a financial or personal relationship with other people or organizations that could inappropriately influence or bias the content of the paper.

\section{References}

American Kennel Club, 2006. The complete dog book. 20th ed. New York: Ballantine Books.

Causmaecker V., Daminet S., Paepe D., 2009. Diabetes ketoacidosis and diabetes ketosis in 54 dogs: a retrospective study. Vlaams Diergeneeskundig Tijdschrift 78, 327-337.

Cook A.K., Breitschwerdt E.B., Levine J.F., Bunch S.E., Linn L.O., 1993. Risk factors associated with acute pancreatitis in dogs: 101 cases (1985-1990). Journal of the American Veterinary Medical Association 203, 673-679.

Hess R.S., Saunders H.M., Van Winkle T.J., Shofer F.S., Washabau R.J., 1998. Clinical, clinicopathologic, radiographic, and ultrasonographic abnormalities in dogs with fatal acute pancreatitis: 70 cases (1986-1995). Journal of the American Veterinary Medical Association 213, 665-670.

Hess R.S., Kass P.H., Shofer F.S., Van Winkle T.J., Washabau R.J., Evaluation of risk factors for fatal acute pancreatitis in dogs. Journal of the American Veterinary Medical Association 214, 46-51.

Kluger E.K., Malik R., Ilkin W.J., Snow D., Sullivan D.R., Govendir M., 2008. Serum triglyceride concentration in dogs with epilepsy treated with phenobarbital or with phenobarbital and bromide. Journal of the American Veterinary Medical Association 233, 1270-1277.

Lem K.Y., Fosgate G.T., Norby B., Steiner J.M., 2008. Associations between dietary factors and pancreatitis in dogs. Journal of the American Veterinary Medical Association 233, 1425-1431.

Mehler S., Bennet R., 2006. Canine extrahepatic biliary tract disease and surgery. Compendium on Continuing Education for Veterinarians 4, 302-314. 
Newman S., Steiner J., Woosley K., Barton L., Ruaux C., Williams D., 2004. Localization of pancreatic inflammation and necrosis in dogs. Journal of Veterinary Internal Medicine 18, 488493.

Newman SJ, Steiner JM, Woosley K, Williams D.A., Barton L., 2006. Histologic assessment and grading of the exocrine pancreas in the dog. Journal of Veterinary Diagnostic Investigation 18, 115-118.

Steiner J.M., Xenoulis P.G., Anderson J.A., Barr A.C., Williams D.A., 2008. Serum pancreatic lipase immunoreactivity concentrations in dogs treated with potassium bromide and/or phenobarbital. Veterinary Therapeutics 9, 37-44.

Watson P.J., Roulois A.J., Scase T., Johnston P.E., Thompson H., Herrtage M.E., 2007. Prevalence and breed distribution of chronic pancreatitis at post-mortem examination in firstopinion dogs. Journal of Small Animal Practice 48, 609-618.

Watson P.J., Archer J., Roulois A.J., Scase T.J., Herrtage M.E., 2010a. Observational study of 14 cases of chronic pancreatitis in dogs. Veterinary Record 167, 968-976.

Watson P.J., Roulois A.J., Scase T.J., Irvine R., Herrtage M.E., 2010b. Prevalence of hepatic lesions at post-mortem examination in dogs and association with pancreatitis. Journal of Small Animal Practice. 51, 566-572.

Watson P.J., Roulois A., Scase T., Holloway A., Herrtage M.E., 2011. Characterization of chronic pancreatitis in English Cocker Spaniels. Journal of Veterinary Internal Medicine 25, 797804.

Witt H., Apte M.V., Keim V., Wilson J.S., 2007. Chronic pancreatitis: challenges and advances in pathogenesis, genetics, diagnosis, and therapy. Gastroenterology 132, 1557-1573.

Xenoulis P.G., Suchodolski J.S., Steiner J.M., 2008. Chronic pancreatitis in dogs and cats. Compendium on Continuing Education for Veterinarians 30, 166-181.

Xenoulis P.G., Steiner J.M., 2010. Lipid metabolism and hyperlipidemia in dogs. The Veterinary Journal 183, 12-21.

Xenoulis P.G., Suchodolski J.S., Ruaux C.G., Steiner J.M., 2010. Association between serum triglyceride and canine pancreatic lipase immunoreactivity concentrations in miniature schnauzers. Journal of the Animal Hospital Association 46, 229-234.

Xenoulis P.G., Levinski M.D., Suchodolski J.S., Steiner J.M., 2011. Serum triglyceride concentrations in Miniature Schnauzers with and without a history of probable pancreatitis. Journal of Veterinary Internal Medicine 25, 20-25. 